\title{
Becoming Cyborg: \\ Interdisciplinary Approaches for Exoskeleton Research
}

\author{
Elizabeth Jochum Aalborg \\ University Rendsburggade 14, \\ 9000 Aalborg, Denmark \\ jochum@hum.aau.dk \\ Louis-Philippe Demers Nanyang \\ Technological University 50 \\ Nanyang Ave, Singapore \\ 638798 Ipdemers@ntu.edu.sg
}

\author{
Bill Vorn Concordia University \\ 1515 St. Catherine W. Montreal, \\ Quebec, Canada \\ bill.vorn@concordia.ca
}

Evgenios Vlachos University of Southern Denmark Campusvej

55, Odense, Denmark

evl@bib.sdu.dk

\author{
Paul Mcllvenny Aalborg \\ University Kroghstraede 3 \\ Aalborg, Denmark \\ paul@cgs.aau.dk
}

\author{
Pirkko Raudaskoski Aalborg \\ University Rendsburggade 14 \\ Aalborg, Denmark \\ pirkko@hum.aau.dk
}

\begin{abstract}
This paper describes novel interdisciplinary approaches for the design and training of cyborg technologies, specifically upper body exoskeletons. EXACT: Exoskeletons, Art and Choreographic Training is a multi-faceted research effort that uses dance performance and experimental trials to study the effects of movement and live performance on exoskeleton training. The goal is to combine research methods from the arts with human robot interaction (HRI) research. The rationale for using ethnographic methods (which privilege qualitative analysis through video data and multimodal interaction analysis) within an HRI framework is to develop nuanced approaches for studying embodiment and techno-corporeality in socially-situated contexts. This investigation has led to the development of new evaluation tools and frameworks for studying human-machine interaction, including human-centred assessments and custom virtual reality tools that allow for fine-grained analysis. An interdisciplinary approach is essential for studying the corporeal experience in human-machine interactions.
\end{abstract}

Exoskeletons, Cyborg, Embodiment, Human Robot Interaction, Inter-corporeality, Dance, Multimodal interaction.

\section{INTRODUCTION}

The cyborg figure in art, science fiction and popular culture typically evokes a dystopian future where humans are enslaved to machines. Donna Haraway famously reframed the cyborg as a positive, agile metaphor for rethinking women's relationship to technology (Haraway 1991). Increasingly, figural and metaphorical cyborgs are confronted with realworld cyborgs in the form of exoskeletons and intelligent prosthetics. The term cyborg is used to describe actual human bodies augmented with smart prosthetics or wearable robots that produce symbiotic, human-machine hybrids. As wearable robots become commonplace in rehabilitation (Zhou et al. 2017), manufacturing (Thilmany 2017), and in private homes (Bai et al. 2017), it is worthwhile considering how alliances between humans and machines come into being. Advanced robotics and automation technologies are rapidly transforming labor practices across many sectors (Pham et al. 2018); this shift has important consequences for human labor and raises broader questions about the socio-cultural and psychological impact of these technologies. This paper advocates for a critical study of cyborgs and human-machine systems through the close examination of specific interactions and situated experiences with wearable 
robots. Material cyborgs are ideal phenomena through which we can examine the complexity and contingencies of human-machine interactions.

In this paper we outline a novel, interdisciplinary research project that introduces artistic and ethnographic methods into the design and research of cyborg technologies, specifically upper body exoskeletons. EXACT: Exoskeletons, Art and Choreographic Training uses dance performance and laboratory trials to study the effects of movement and live performance on exoskeleton training. Our goal is to combine research methods from the arts and social sciences to advocate for a human-centred approach to exoskeleton research. Central to our inquiry are the concepts of technocorporality and relationality: how humans experience movement inside an exoskeleton.

Exoskeletons rely on intimate, physical alliances with human bodies. Like the example of the blind person's stick, wearable robots are assimilated and become part of the human body through motility, transforming sensory perceptions and subjective experience of the body in the world. Exoskeletons instantiate the techno-corporeal paradox of embodied movement: the human body is simultaneously master and slave, agent and object, in a transgressive assemblage that is enacted in a relational process of becoming. Becoming-cyborg is not a transformation from human to machine; it is a relational becoming. Becoming-cyborg does not occur simply by donning a costume or prosthetic, but emerges through continuous and evolving corporeal experience of embodied movement.

The qualitative experience of movement-how well a patient moves with a prosthetic or how comfortably an exoskeleton facilitates a walking motion-has a profound impact on the acceptance and success of exoskeletons and other human-machine systems. However, capturing the human experience of movement is a notoriously elusive task, and very difficult to represent through language. Working with an interdisciplinary team of artists and researchers from $\mathrm{HCl}, \mathrm{HRI}$ and the visual and performing arts, we designed a research strategy based on an interactive dance performance. Our aim is to recover the qualia that is often overlooked in the study of human-machine systems by creating new assessment tools that allow researchers to study human-machine interaction from a human-centered perspective.

The paper is organized as follows: we first introduce the concept of performance as research and describe the performance context. Section 3 introduces the concept of becoming as it relates to the corporeal turn and the study of movement. Section 4 describes the research design and experimental set-up. In Section 5, we present the methods and custom evaluation tools we developed to carry out the study. The ethnomethodological approach and tools for evaluating embodied experience are novel contributions of this study. Section 6 includes our preliminary findings, and we conclude with a brief discussion of the advantages of interdisciplinary research for studying humanmachine systems from a human-centered perspective.

\section{Performance AS ReSeARCH}

The arts have long been fertile ground for exploring the human fascination and fear surrounding technology. Artistic inquiry opens up new pathways for engaging with technologies while simultaneously interrogating what these technologies might mean for society. Often, art works can challenge conventional notions of how we experience, make sense of, and relate to human experiences in an increasingly technologized world. Among the arts, live performance is one of the most dynamic spaces for exploring transgressive technologies because it allows for possibilities to emphasize or reconceptualize the human body's relationship to technology. In the essay Becoming Animate, Jennifer Parker-Starbuck proposes performance as a kind of experimental laboratory for experiencing and exploring becoming. Performance creates ethical spaces for the examination of humans and technologies, and can inspire audiences to "reconsider the impact, coexistence, and interdependency between technologies, humans, and animals" (Parker-Starbuck 2006, 651-652).

Theatre and live performance have proven to be valuable sites for conducting tractable HRI research. Scripted theatre plays can be used as discussion tools concerning possible future care and work scenarios between humans and robots (Walters et al. 2013; Jochum et al. 2017), or as sites for exploring innovative approaches to the design and control of creative machinic performers and their effects on audiences (Demers 2016; Jochum et al. 2016; Vorn 2016). Typically, audiences observe a performance and afterward complete selfassessments or group interviews. Building on previous work that used artistic venues to study HRI (Vlachos et al 2016), we formulated a research plan to obtain data on corporeal experience of humanmachine interaction. Our research is guided by the notion that live performance can support training of human-machine configurations in non-performance settings.

EXACT centers around the live, interactive, participatory dance performance Inferno developed by Louis-Philippe Demers and Bill Vorn (Demers and Vorn 2018). The performance consists of a set of twenty-four upper body exoskeletons (the actual number varies according to the venue) that actively manipulate and control the participants' motions 
according to a pre-programmed score and choreography (Figure 1). The performance lasts 60 minutes, during which the participants (audience members) are guided through a series of choreographic motions that are synchronized with special effects such as music and lighting cues. At selected moments in the performance, the prerecorded sequences are interrupted and the choreography is tele-operated, either by operators controlling the motions directly through telematic devices, or by a custom exoskeleton worn by a performer who controls the motions of the other exoskeletons in real-time. The open-ended nature of the performance is designed to encourage playfulness and facilitate spontaneous movement by the participants.

Demers and Vorn's previous collaborative art works include interactive robotic installations ( $L a$ Cour des Miracles, 1997; Lost Referential, 1998), and live performance (Le Procès, 1999), as well as individual projects such as the Blind Robot (Demers 2012) and Hysterical Machines (Vorn 2002-2004). Inferno goes a step further in exploring human-machine interaction. Whereas previous works utilised professional human performers alongside robot performers, there are no professional performers in Inferno and there is no prior training that prepares participants to use the technology. Instead, general audience members don exoskeleton suits and become the performers. The direct physical involvement of the audience creates the possibility for studying human-machine interaction.

When touring the performance, Demers and Vorn observed literally hundreds of novices learning to move in harmony with exoskeletons. For each performance, the artists observed a detectable moment when audiences would "give over to the machine." According to Demers, this signalled the moment when participants begin working with the prescribed motions instead of against them. The artists were interested in finding out more about what precisely was happening at that moment, and whether this shift could be detected through documentation or movement analysis.

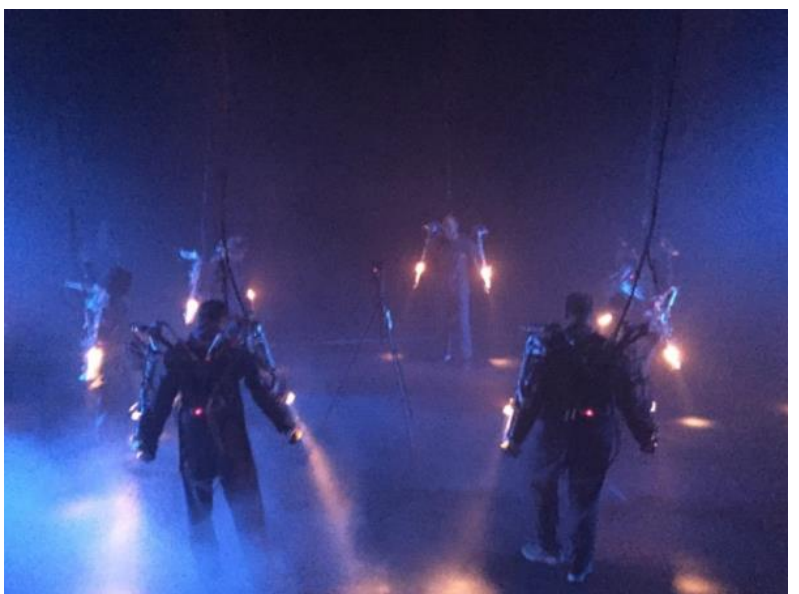

Figure 1: Inferno (Louis-Philippe Demers and Bill Vorn) is an interactive dance performance where audiences perform wearing tele-operated upper body exoskeletons.

Inferno is an example of a class of robot and cyborg performance that Steve Dixon terms "Metal Performance," where "metallic" denotes not only the physical material but also cultural connotations that signal loud, aggressive, and resistance expression (Dixon 2004, 16). To a great extent, Inferno capitalizes on human fears and fascination with machinic embodiments, and explores the thrill and fear of the humanization of machines and the dehumanization (or "machinization") of humans. Inferno leverages the dystopian master-slave trope typical to cyborgs in art and science fiction, creating a dramatic effect for the audience. Paradoxically, while the aesthetic experience is predicated on fear and loss of control, the actual human-machine interaction is profoundly pleasurable. Vorn and Demers observed that, although unfamiliar and slightly afraid of the technology, audiences generally enjoy the experience of being controlled and voluntarily engage in exploratory movements and gestures that go beyond the original choreography. The performance can be read as a kind of parodic irony or mimicry reminiscent of drag, where the mimicry is forced upon the participants audiences through an external costume and scenic conventions.

To understand how Inferno performance constitutes becoming cyborg (and not merely figural cyborgs), we can look to phenomenology and the corporeal turn in social research. The premise of the corporeal turn is that "bodies do not remain fixed or static but are mediated by processes and practices that produce dynamic points of intersection and connection" (Blackman 2008, 107). EXACT is about understanding how these processes and practices are enacted in human-machine interaction. The results have to many efforts and the development of new evaluation tools and frameworks, including a robust assessment criteria and custom VR tools that account for human-machine interaction in situated contexts (discussed in Section 5). The experience 
in Inferno enables a heightened perception of movement while both expanding and limiting opportunities for physical motion. The key to understanding the experience of becoming cyborg lies in addressing this paradox.

\section{BeComing Cyborg}

The concept of "becoming" (first set forth by Deleuze and Guattari, 1987) has been taken up by feminist theory and others interested in the corporeal turn. Together with phenomenology and kinesthetic consciousness, becoming offers a framework for thinking about how bodies are produced and performed in specific social, cultural and material contexts. In short, the question is no longer about "what the body is" but rather about "what a body can do." (Manning in Blackman 2008, 105). While exoskeletons change the physical appearance of the body, they also change what that humanmachine body can do.

Exoskeletons require the ability to coordinate physical actions and stimulate a heightened awareness of how human beings experience themselves as movers. For all animate bodies, perception and action are closely linked. Phenomenologists consider movement the primary means for knowing and reasoning about the world. Maxine Sheets-Johnstone uses the term "creaturely movement" to describe how embodied movement contributes to the experience of aliveness:

[C]reaturely movement, being itself a creature-perceived phenomenon, is in and of itself a form of knowledge. Not only is our own perception of the world everywhere and always animated, but our movement is everywhere and always kinesthetically informed. (SheetsJohnstone 2011, 113).

In her view, the significance of movement for perception has been eclipsed by research grounded in visual perception, language, informationprocessing, and computational modelling. A phenomenologist approach that directs attention back to the actual experience of movement for the human subject is critical for reasoning sensemaking. We define human-centered approaches as those that value the actual experience of real humans. Thus, the corporeal turn can help open up new directions in exoskeleton research.

In phenomenology, the human experience of movement and animacy is tied to spontaneity and exploratory movement. For Sheets-Johnstone, movement constitutes a knowing which forms the epistemological foundation of all learning: humans come to know themselves through movement with respect to objects. It follows that movement can be understood as
"the foundation of our sense of who and what we are. We literally discover ourselves in movement. We grow kinetically into our bodies. In particular, we grow into those distinctive ways of moving that come with our being the bodies we are. In our spontaneity of movement, we discover arms that extend, spines that bend, knees that flex, mouths that shut, and so on. We make sense of ourselves in the course of moving. We discover ourselves as animate organisms." (Sheets- Johnstone 2011, 116).

Exoskeletons paradoxically both impede and facilitate spontaneous movement, creating heightened kinesthetic awareness while simultaneously rendering the body and even basic movements unfamiliar. Moving-and learning to move-with exoskeletons is a lesson in thinking through the device. Scientific inquiry into humanmachine interacti must account for this paradox.

In Inferno, exoskeletons facilitate becoming cyborg through the close, intimate coupling of flesh and machine. Unlike other performances where audiences are positioned at a safe distance from the stage, Inferno requires audiences to enact the performance. Audiences become figural cyborgs when they don the exoskeletons, but the exoskeletons are more than costumes or tools. Through performance, they materially instantiate issues of domination and resistance central to "high technology" and scientific culture. Audiences actively experience the paradox of moving with an exoskeleton; they become more aware of their movements while at the same time their subjective experience of the body in motion is defamiliarized.

The human-machine interaction links to what Rosi Braidotti calls the "paradox of techno-corporeality", where "the corporeal site of subjectivity is simultaneously denied, in a fantasy of escape, and strengthened or reinforced" (Braidotti 2012, 170). Exoskeletons transform the material body and the physical experience associated with human movement, paradoxically expanding the body's capacity for movement while at the same time constricting its function and overall freedom. Exoskeletons and other cyborg technologies offer the fantasy of transcending the natural body through augmentation, but the material body remains tightly bound to the machine, assuming its form, functionality and limitations. Exploring this paradox is the artistic core Demers and Vorn's art work. It is also relevant to the research design for EXACT.

\section{Research Design}


EXACT is a multi-faceted research effort that uses dance performance and experimental trials to study the effects of movement and live performance with exoskeletons. We combine research methods from the arts with human robot interaction (HRI) research. The rationale for using ethnographic methods is to put human experience at the center of the research. We developed a research design that would allow us to leverage the conventions of live performance while still maintaining a rigorous experimental protocol that would satisfy exoskeleton researchers. Our project required that we develop new approaches for studying embodiment and technocorporeality in socially-situated contexts.

Conventional exoskeleton research takes affective experience and the human body for granted. Indeed, the term "human-centered" approach in exoskeleton research refers only to computer models of the human muscular skeletal system in simulation, and not actually to the use of humans in the design process. Following Blackman and others (Barad 2003; Suchman 2007; Sheets-Johnstone 2011), we proceed from the assumption of the "permeability of boundaries and the inextricable connection of mind with body, human with nonhuman and biological with social" (Blackman 2008, 58).

The research design proceeds from the following four arguments: (1) Embodied movement is strongly linked with cognition; (2) Movement as experienced is epistemically different than movement as measured; (3) Linguistic accounts and quantitative measurements of movement alone are insufficient for representing the proprioceptive and kinaesthetic experience of movement; and (4) the study of human-machine interaction must account for the socially-situated contexts where these interactions take place.

Our interest in studying human movement with exoskeletons led to the following research questions:

1) What methods can best prepare a person to use an exoskeleton?

2) What is a useful quantitative measure for analysing the quality of the humanexoskeleton interaction from a human perspective?

These two questions guided the initial round of experiments. We chose to study the effects of live performance in comparison with a laboratory framework. The experiments were conducted over a week-long period and coincided with public performances. We studied two populations in two unique situated contexts: (1) we recruited participants from the community for the experimental trials, and (2) we administered assessment forms to the general audiences who attended the performance without prior knowledge of the research.

Working with two distinct sample populations and contexts (experimental/laboratory and performance) enables us to evaluate the effects of the sociallysituated contexts on the outcomes. From an ethnographic perspective, the laboratory and theatre space are socially and culturally charged spaces that produce different meanings and configurations. The paradigms and modes of interaction shift depending on context, and we anticipated this discrepancy would impact the findings. The openended, playful and communal aspects of a group performance in the presence of a live audience is dramatically different than a laboratory setting, where individual study participants test exoskeleton under close observation of a team of researchers.

All study participants were novices (untrained users), and had no prior experience with exoskeletons (nor had any participated in a prior Inferno performance). Condition 1 simulated a laboratory scenario where the participants were given a set of choreography lasting about eight minutes, and after a short break they repeat the same choreography [Figure 3]. For Condition 2, the only change was the addition of lights and sound (e.g. the same performance conditions as in the Inferno performance, but without an audience). For Condition 3, participants followed the same procedure as Condition 2, but prior to the trial they participated in a half-hour lesson on contact improvisation led by a professional choreographer and dance instructor. Contact improvisation is an experimental method for generating improvised performances or generating movement material. Dancers engage in guided exercises and encouraged to experiment with principles of motion and balance [Figure 4]. Participants explored physical sensations of movement with eyes closed and through touch. The exercises were selected to stimulate a heightened awareness of the physical body, and to become familiar and responsive to other bodies through physical exercises. Neither exoskeletons nor robots were mentioned during the session. 


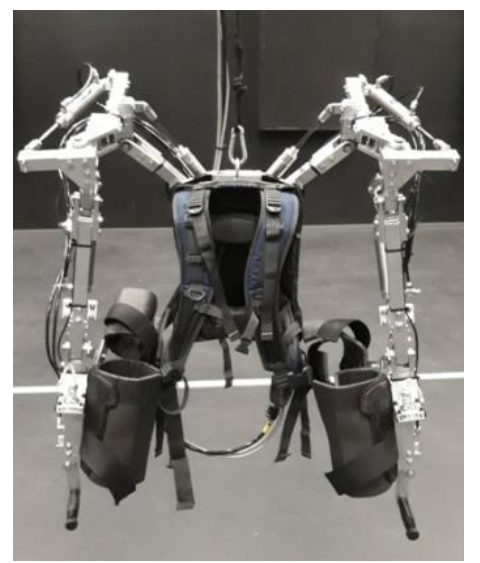

Figure 2: The upper-body exoskeleton used in Inferno. During the performance, the participant carries the entire weight of the machine (c. 13 kilograms).

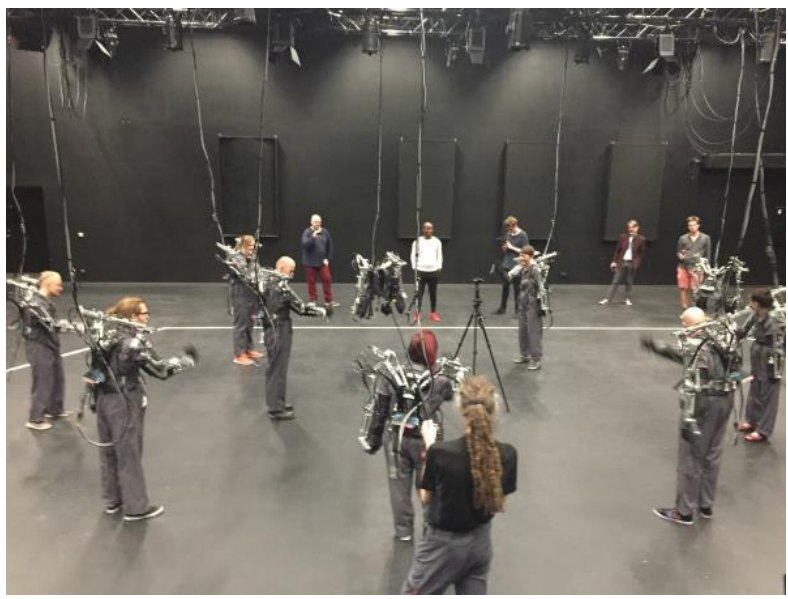

Figure 3: The experiments were conducted in a theatre and simulated a laboratory scenario. A team of researchers recorded the trials, which included dressing, a set of choreography lasting eight minutes, and a follow up focus group interview.

We worked with a combination of quantitative and qualitative methods that captured the individual experiences of study participants. This included a team of video researchers and the development of a subjective, self-assessment measurement inspired by the Functional Movement Screen (Cook 2006). Together, these assessment tools provide methods for analyzing the conditions of the experimental set up as well as the qualitative experience of the participants. In the next section we describe the key aspects of these tools, and outline their potential for further development.

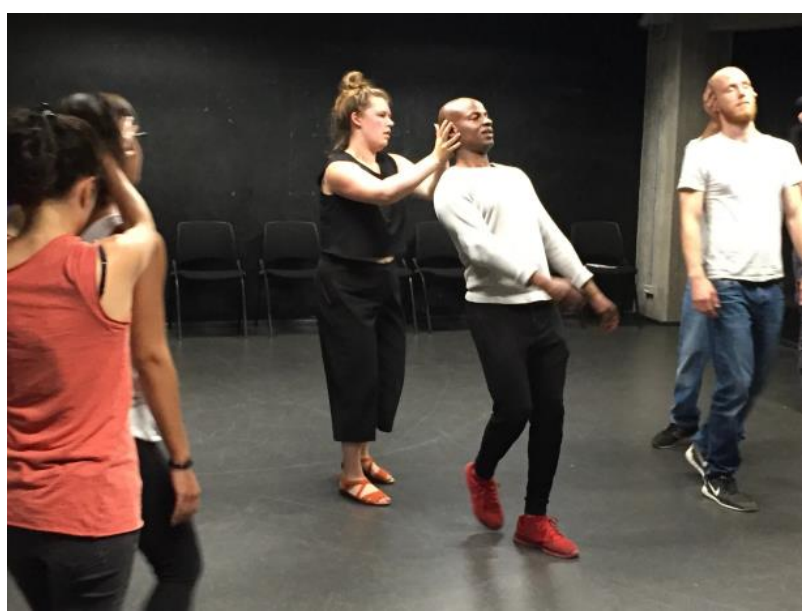

Figure 4: In Condition 3, participants were led in a halfhour session of contact improvisation, an experimental dance technique where human dancers experiment with motion and balance.

\section{New Qualitative Tools}

The qualitative analysis of exoskeleton performance provides a nuanced understanding of the issues and challenges related to conducting experiments with wearable robots and highlights how artistic methods can be used to conduct and HRI research. Thus far, EXACT researchers have developed three new tools for assessing the qualia of human experience of motion. These tools are efforts to place the human at the center of the study, and to evaluate the interactions not as fixed but rather as a relational becoming. After all, "the most innovative setup is based on analysable sensibilities" (Raudaskoski and Mitchell 2013).

\subsection{HARMONY EXoskeleton Self-Assessment TEST (HEAT)}

Reviewing assessment tools for exoskeletons in scientific research, we were surprised by the lack of established assessment criteria or benchmarks for evaluating exoskeletons. A literature review revealed that measurements rely predominantly on quantitative measurements related to taskperformance, effort and fatigue. Furthermore, nearly all exoskeleton trials are conducted in laboratory settings that do not address the complex materiality and socially-situated nature of the experiments. To develop a human-centred and contextualized assessment tool, we formulated and tested the validity and reliability of the Harmony Exoskeleton Self-Assessment Test (HEAT). HEAT is a subjective measurement tool that assesses an individual's experience of movement in an exoskeleton, based on a numerical score (Vlachos et al. 2018). The test asks participants to evaluate their experience of movement on six factors: fatigue, 
coordination, balance/body control, ease of movement, progress of movement, and ease of use. Each factor consists of four possible scores ranging from 0 to 3, with zero being the worst score, and each score represents a state. The maximum score that can be achieved is 18 , and the lowest is 0 . HEAT is designed to be adaptable for, and ideally used as an assessment tool for evaluating holistic interaction experiences with exoskeletons. The average HEAT score for the particular Inferno performance was 13.3 out of 18 , signifying a rather harmonious coexistence of the participants with the heavy, non-compliant exoskeletons.

\subsection{SQUIVE \& AVA360VR}

In this project, the ethnographic methods for the study of movement rely on video-based inquiry. Techniques for qualitative inquiry can include video ethnography, performance documentation, and multimodal interaction analysis. The possibilities for comprehensive video capture have expanded as a result of affordable and commercially available tools such as 360-degree cameras, depth mapping sensors, ambisonic sound, and software tools that enable multi-camera video stitching and manipulation. Working with these tools, EXACT researchers are developing advanced approaches under the auspices of Big Video (Mcllvenny and Davidsen 2017). Big Video focuses on complex human data capture scenarios, such as the INFERNO performance and EXACT trials, to develop new approaches to documentation and new qualitative analytical tools for video research [Figures 5-7]. The scenographic approach involves recording and analysing video data to document rich, complex scenarios such as the dance performance and experimental trials. Novel tools and interdisciplinary approaches are developed in order to address immersive and embodied interactive cyborg experience by documenting the specificity of the site.

Two key concepts inform the design of two software prototypes to facilitate qualitative analysis. First, SQUIVE (Staging QUalitative Immersive Virtualization Engine) is based on the principle of staging video, which involves reconstructing the site and the scenes in which cyborg interactions took place over time in an interactive and immersive 3D representation in mixed reality video (Figures 6-7). Second, AVA360VR (Annotate, Visualize, Analyze 360 -degree video in VR) is based on the principle of inhabiting video to facilitate exploring complex spatial video and audio recordings of a single scene in which cyborg interaction took place through a tangible interface in virtual reality (Mcllvenny 2018). These tools provoke new insights into relevant concepts for the study of movement, including spatiality, mobility, volumetricity, intercorporeality and materiality.

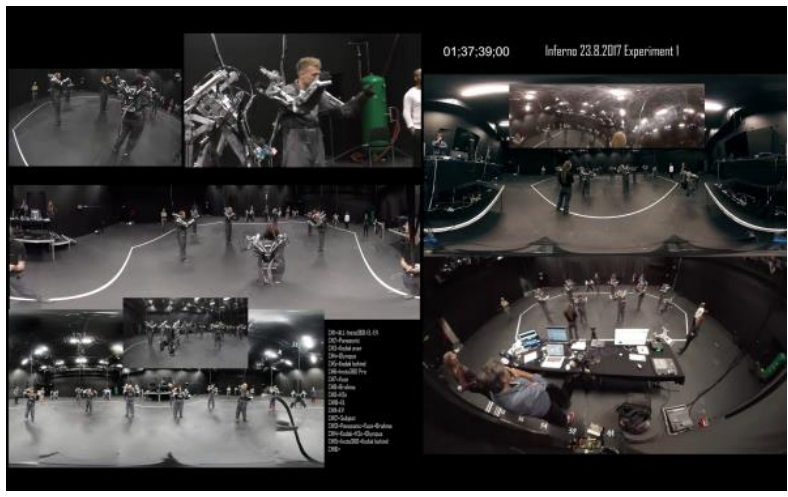

Figure 5: EXACT uses multiple cameras and ambisonic sound recording equipment to develop custom software tools for multimodal interaction analysis research in 3D environments.

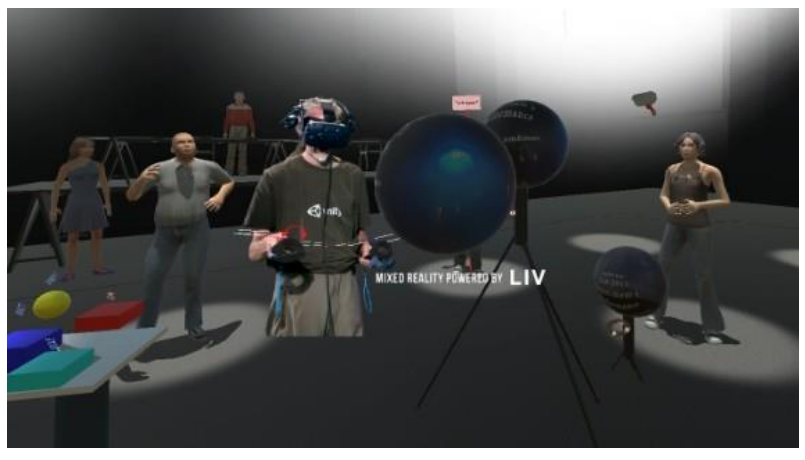

Figure 6: A screenshot of the virtual researcher walking through and manipulating the $3 \mathrm{D}$ reconstruction of the experiment, which includes avatars, the virtual camera locations and actual 360-degree video footage (spheres).

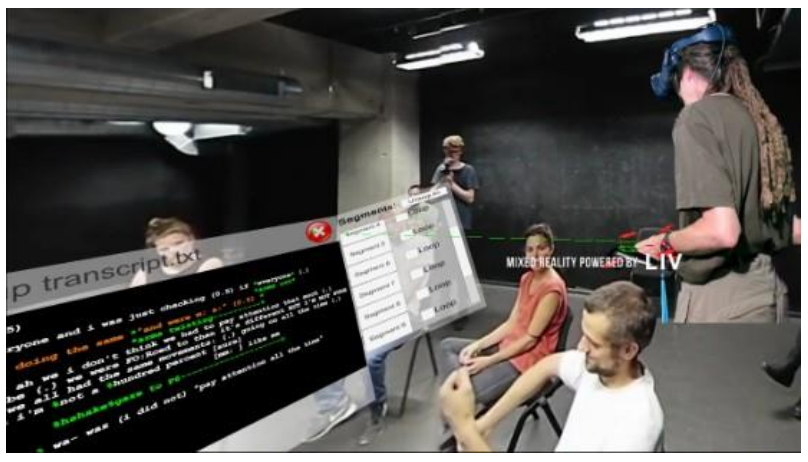

Figure 7: A screenshot of the virtual researcher working with a transcript of talk in the Condition 3 focus group in which a subject (below) re-enacts his shared embodied experience with the exoskeleton.

\section{Preliminary Findings}

The ethnographic studies conducted demonstrate that linguistic accounts of movement are insufficient for representing the unique, qualitative experiences 
that participants in the INFERNO performances and EXACT trials have of their cyborg embodiment, mobility and motility. Across all three experimental groups, the subjects verbalise their experiences with their cyborg embodiments as alienating with a loss of agency, and yet they recovered their own sense of control and embodiment from observing the others (Figure 6). For example, one subject said, "I actually focused on the others just to see what am I doing right now." This result could be seen as evidence for the participants' learning to give in to new experience(s). In Blackman's words, "learning is not a cognitive skill developed and undertaken by a brain or mind, but rather denotes the capacity of bodies to acquire more and more connections to artefacts, techniques and practices" (Blackman 2008, pp. 106). This remark from a participant offers empirical evidence to the idea that "the body is never a singular body but a complex relational process" (Blackman 2008, 81). Although there are clear similarities across the groups, qualitative analysis shows that the preparatory dance workshop for Condition 3 was an important factor in the familiarisation of the subjects with their exoskeletons and their subsequent improvisatory movements. This corroborates the results of the quantitative study, which we plan to study in future work.

\section{CONCLUSION}

Advancements in robotics, biomechanics and neuroscience have led to medical breakthroughs in smart, assistive prosthetics and devices. Wearable robots are increasingly part of work, home, and health environments. As with all emerging technologies, wearable robots will transform human labor practices with consequences. These cyborg technologies give rise to important ethical and humanitarian considerations; therefore robust frameworks that address human well-being and societal impact should be prioritized at every level of research and development. One way to address this complexity is to adopt human-centred approaches to exoskeleton research that accounts for embodied and subjective experiences.

In this paper we outlined novel interdisciplinary approaches for the study of exoskeletons from a human-centred perspective. Traversing different modes of thinking has important consequences for scientific claims and research outcomes: our hope is that human-centered and interdisciplinary approaches might contribute to strong objectivity and prevent re-inscribing unquestioned gender and political assumptions that inform the practice of science. For, as Karen Barad observes

'Humans' do not simply assemble different apparatuses for satisfying particular knowledge projects but are themselves specific, local parts of the world's ongoing reconfiguring. To the degree that laboratory manipulations, observational interventions, concepts, or other human practices have a role to play is as part of the material configuration of the world in its intra-active becoming. (Barad 2003, 829)

Studies grounded in corporeal experience have the potential to expose some of the scientific and cultural assumptions that underlie research and development particular to exoskeletons. The rationale for using ethnographic methods within an $\mathrm{HRI}$ framework is to develop nuanced approaches for studying embodiment and techno-corporeality in socially-situated contexts.

\section{ACKNOWLEDGEMENTS}

Inferno is designed, choreographed, composed and realized by Louis-Philippe Demers and Bill Vorn, and was produced with help from the Canada Arts Council and a Ministry of Education Grant (Tier1) from Singapore. The premiers of Inferno was coproduced by Elektra and Arcadi. The authors wish to thank Teater Nordkraft where the EXACT experiments took place. We also thank Todd Murphey and Raj Madhavan for helpful discussions.

\section{REFERENCES}

Bai, S., Christensen, S., Islam, M.R.U. (2017). An upperbody exoskeleton with a novel shoulder mechanism for assistive applications. IEEE International Conference on Advanced Intelligent Mechatronics, Munich, Germany , 3-7 July 2017, 1041-1046. AIM, Germany.

Barad, K. (2003) Posthumanist Performativity: Toward an Understanding of How Matter Come to Matter. Signs: Journal of Women and Culture in Society 8(3), pp. 801831.

Blackman, L. (2008). The Body. Oxford: Berg.

Braidotti, R. (2012). Afterword: Complexity, materialism, difference. Angelaki, 17:2, pp. 169-176.

DIGHUMLAB. https://dighumlab.org (Retrieved 14 June 2018).

DeLeuze, G. and Guattari, F. (1987) A Thousand Plateaus. Trans. Massumi, B. University of Minnesota.

Demers, L-P. and Vorn, B. (2018) Performing the Machine: the Inferno Paradox. Electronic Visualisation and the Arts: Politics of the Machine (EVA2018), Copenhagen, DK, 15-17 May 2018. EVA, Copenhagen.

Demers, L-P. (2016) The Multiple Bodies of a Machine Performer. In Herath, Kroos and Stelarc (eds.) Robots and Art, Springer, Singapore. 
Dixon, S. (2004). Metal Performance: Humanizing Robots, Returning to Nature, and Camping About. The Drama Review, 48(4), pp. 15-46.

Haraway, Donna J. (1991). Simians, Cyborgs, and Women. London, Press Association Books.

Inferno (2015) by Louis-Philippe Demers and Bill Vorn. Permanent link available at http://vimeo.com/lpdemers/Inferno2017 (retrieved 11 June 2018).

Jochum, E., Vlachos, E., Chistoffersen, A., Nielsen, S.G., Hameed, I.A., Tan, Z.-H.. (2016) Using Theatre to Study Interaction with Care Robots. International Journal of Social Robotics (8), pp. 457-470.

Jochum, E., Millar, P., and Nuñez, D. (2017) Sequence and Chance: Design and Control methods for Entertainment Robots. Robotics and Autonomous Systems 87, pp. 372-380.

Kim, B., Deshpande, A. (2017) An upper body rehabilitation exoskeleton Harmony with an anatomical shoulder mechanism: Design, modelling, control, and performance evaluation. International Journal of Robotics Research 36(4), pp. 414-435.

Mcllvenny, P. (2018) Doing Being a Cyborg: Robot Exoskeletons and Re-Enactments of Intercorporeality. International Conference on Conversational Analysis (ICCA) Loughborough, UK, 11-15 July 2018. ICCA, Loughborough.

Mcllvenny, P., Davidsen, JR. (2017) A Big Video Manifesto. Nordicom-Information 39(2), pp. 15-21.

Parker-Starbuck, J. (2006). Becoming-Animate: On the performers limits of "Human". Theatre Journal 58(4), pp. 649-668.

Pham, Q.C., Madhavan, R., Righetti, L., Smart, W., and Chatila, R. (2018) The Impact of Robotics and Automation on Working Conditions and Employment. IEEE Robotics \& Automation Magazine, pp.126-128.

Raudaskoski, P. and Mitchell, R. (2013) "The situated accomplishment (aesthetics) of being a cyranoid." In Participatory Innovation Conference 2013. Ed. Helinä Melkas and Jacob Buur. Lappeenranta University of
Technology Scientific and Expertise Publications 6, pp. 126-129.

Rothfield, P. (2008) Philosophy and the Bodily Arts. Parallax, 14(1), pp. 24-35.

Sheets-Johnstone, M. (2011) Primacy of Movement. John Benjamins Publishing Company, pp .113-152.

Suchman, L. (2007) Human-Machine Reconfigurations. (Plans and Situated Actions, 2nd Edition). Cambridge, Cambridge University Press.

Thilmany, J. (2017) Robotic Suits May Transform Manufacturing. American Society for Mechanical Engineers (ASME). www.asme.org (retrieved 11 June 2018).

Turkle, S. (2008) The Inner History of Devices. Cambridge, MIT Press.

Vernon, D. (2014) Artificial Cognitive Systems. Cambridge, MIT Press.

Vlachos, E., Jochum, E., and Demers, L-P (2016) The Effects of Exposure to different social robots on attitudes towards preferences. Interaction Studies 17, pp. 390404.

Vlachos, E., Jochum, E., and Demers, L-P (2018) "HEAT: The Harmony Exoskeleton Self-Assessment Test. IEEE International Symposium on Robot and Human Interactive Communication (RO-MAM), Nanjing, China, 27-31 August, pp. 577-582.

Vorn, B. (2016) I Want to Believe-Empathy and Catharsis in Robotic Art. In Herath, Kroos and Stelarc (eds.) Robots and Art, Springer, Singapore.

Walters, M., Koay, M.L., Syrdal, D.S., Cambpbell, A. Dautenhahn, K. (2013) Companion Robots for Elderly people: Using theatre to investigate potential users' views. IEEE International Symposium on Robot and Human Interactive Communication (RO-MAN), pp. 691696.

Zhou, L., Li, Y., and Bai, S. (2017) A human-centered design optimization approach for robotic exoskeletons through biomechanical simulation. Robotics and Autonomous Systems 91(C), pp. 337-347. 